\title{
Comparação entre turbinoplastia e turbinectomia endoscópicas: Eficácia e parâmetros clínicos
}

\section{Comparison between turbinoplasty and endoscopic turbinectomy: Efficacy and clinical parameters}

\author{
Marcos Marques Rodrigues', Ralph Silveira Dibbern², Luis Francisco de Oliveira ${ }^{3}$, Melania Dirce Oliveira Marques ${ }^{4}$, \\ Marcelo Fernando Bella 5 , Fausto Antonio de Paula Junior ${ }^{3}$, Fernando Cesar França Araújo4. \\ 1) Professor \\ 2) Mestre em Otorrinolaringologia pela Faculdade de Medicina da USP Ribeirão Preto. Médico Otorrinolaringologista responsável pelo setor de Apneia do Sono do \\ Departamento de Otorrinolaringologia da Santa Casa de Limeira. \\ 3) Médico Otorrinolaringologista da Santa Casa de Limeira. \\ 4) Médico (a) Residente do Departamento de Otorrinolaringologia da Santa Casa de Limeira. \\ 5) Coordenador do Departamento de Otorrinolaringologia da Santa Casa de Limeira. \\ Instituição: Irmandade da Santa Casa de Misericórdia de Limeira. \\ Limeira / SP - Brasil. \\ Endereço para correspondência: Marcos Marques Rodrigues - Avenida Geraldo Hilário da Silva, 146 - Jardim Flamboyant II - Araraquara / SP - Brasil - CEP: 14805 -290 \\ - Telefone: (+55 16) 8108-4840 - E-mail: marcosmmr@hotmail.com \\ Artigo recebido em 10 de Março de 2011. Artigo aprovado em 10 de Julho de 2011
}

\section{RESUMO}

Introdução: Obstrução nasal é um sintoma comum e atinge $25 \%$ da população. A hipertrofia de cornetos inferiores é a principal causa de obstrução nasal. Na falha do controle clínico um procedimento cirúrgico para redução do volume dos cornetos inferiores é indicado.

Objetivo: Comparar a melhora da qualidade de vida no pósoperatório tardio de turbinectomia e turbinoplastia.

Método: Estudo de Série de Casos retrospectivo. Foram avaliados 24 pacientes submetidos à cirurgia nasal de turbinectomia ou turbinoplastia em 2007. Os pacientes foram convocados para uma entrevista em agosto de 2008. Os pacientes foram avaliados quanto aos seguintes itens: escala NOSE pós-operatória, morbidade no $\mathrm{PO}$, sangramentos e quantidade de crostas no PO.

Resultados: Compareceram para a avaliação 24 pacientes. A principal variável analisada foi à diferença entre a escala NOSE no pré e no pós-operatório tardio. Não houve significância estatística pelo teste t de student nas variáveis estudadas.

Discussão: $\mathrm{Na}$ avaliação dos diversos tipos de tratamento cirúrgico do corneto inferior a literatura mostra resultados semelhantes ao do nosso estudo encontrando resultados semelhantes entre as diversas técnicas cirúrgicas quanto à melhora da obstrução nasal e a atividade mucociliar.

Conclusão: Não há evidencia na literatura e em nossa amostra da superioridade de uma técnica do tratamento cirúrgico do corneto inferior sobre os outros tratamentos.

Palavras-chave: obstrução das vias respiratórias, obstrução nasal, rinite.

\section{SUMMARY}

Introduction: Nasal Obstruction is a common symptom and affects $25 \%$ of the population. The inferior turbinate hypertrophy is the main cause of nasal obstruction. In the failure of clinical control, a surgical procedure to reduce the size of the inferior turbinate is indicated.

Objective: Compare the improvement of life quality in late postoperative of Turbinectomy and turbinoplasty.

Method: Study of a retrospective case series. 24 patients were submitted to a nasal surgery of turbinectomy or turbinoplasty in 2007. The patients were invited to an interview in august of 2008. The patients were evaluated in the following items: Postoperative NOSE scale, morbidity in postoperative, bleeding and quantity of crusts in postoperative.

Results: 24 patients attended for the evaluation. The main variable analyzed was the difference between NOSE scales in late pre and post operative. There was no statistically significant by the test in the variables studied. Discussion: In the evaluation of the various types of surgical treatment of the inferior turbinate, literature shows similar results to our study, finding similar results between many surgical techniques in the improvement of the nasal obstruction and in mucociliary activity.

Conclusion: There are no evidence in the literature and in our sample of the superiority of a technique of surgical treatment in the inferior turbinate under other treatments.

Keywords: airway obstruction, nasal obstruction, rhinitis. 


\section{INTRODUÇÃO}

Obstrução nasal (ON) é um sintoma comum e atinge $25 \%$ da população. As principais etiologias para $\mathrm{ON}$ são: desvio septal, hipertrofia de cornetos inferiores e médios, polipose nasal e hipertrofia de tonsilas faríngeas (1). Dentre essas etiologias a hipertrofia de cornetos inferiores (HCCI) figura como a principal causa de obstrução nasal (2). Cerca de 20\% da população europeia tem obstrução nasal crônica causada pela HCCI (3). Rinopatia alérgica, rinite vasomotora e desvio septal (hipertrofia compensatória) figuram como as principais causas de HCCI (4).

Existem várias opções para o tratamento clínico da HCCI. Anti-histamínicos, corticoide tópico nasal e solução salina são as principais drogas utilizadas. Vasoconstritores e injeção de corticoides são usadas em menor escala devido aos potenciais efeitos deletérios (4). Na falha do controle clínico indica-se geralmente um procedimento cirúrgico para redução do volume dos cornetos inferiores. As principais técnicas descritas são: turbinectomia total ou parcial, turbinoplastia, ressecção submucosa, turbinoplastia laserassistida, criocirurgia, tratamento com luz infravermelha, cirurgia de plasma de argônio, aplicação tópica de AgNO3, eletrocautério monopolar e bipolar e redução volumétrica dos tecidos por radiofrequência (5).

A ON é um sintoma que produz importante interferência na qualidade de vida. A obstrução nasal dificulta a qualidade do sono e exercícios físicos, causa cansaço e irritabilidade. Em crianças a patência é fundamental para o desenvolvimento crânio-facial, crianças respiradoras bucais tendem a desenvolver palato ogival, face alongada e retrognatia perpetuando a obstrução nasal nos futuros adultos (6).

A avaliação da qualidade de vida e eficácia de tratamento da ONé difícil. Assima Academia Americana de Otorrinolaringologia validou o questionário intitulada Escala de Avaliação Sintomática de Obstrução Nasal. O nome original da escala é Nasal Obstruction Syndrome Evaluation, conhecida como NOSE scale, termo usado nesse texto. A escala é feita de 5 perguntas sobre a qualidade de vida.
Cada pergunta recebe uma nota que varia de 0 a 4 . Essas notas são somadas e multiplicadas por 20. Dessa forma a escala NOSE varia de 0 a 100 (7). A escala traduzida para o português se encontra na Tabela 1 .

A escala NOSE se tornou a referencia para estudos que analisam a influencia sobre da ON sobre a qualidade de vida, tanto basal quanto no seguimento após tratamentos clínicos e cirúrgicos (8).

O objetivo deste estudo é comparar a melhora da qualidade de vida no pós-operatório tardio de turbinectomia e turbinoplastia.

\section{MÉTODO}

Estudo aprovado pelo Comitê de Ética e Pesquisa da Irmandade da Santa Casa de Misericórdia de Limeira sob o protocolo 145/09 e devidamente registrado no Clincal Trials Registry. Foram avaliados 24 pacientes submetidos à cirurgia nasal de turbinectomia ou turbinoplastia sem septoplastia no período de janeiro a dezembro de 2007. Os pacientes foram submetidos a nasofibroscopia com fibroscópio Machida ENT-30 PIII de 3,2mm foram analisados quanto ao nível de obstrução das vias aéreas superiores. Os pacientes eram portadores de hipertrofia de corneto inferior confirmados pela nasofibroscopia feita com anestésico tópico e sem vasoconstritor para não enviesar a análise da hipertrofia de corneto inferior. Todos os pacientes da amostra eram portadores de rinite alérgica e foram submetidos a tratamento com anti-histamínico oral por 30 dias e corticoide tópico nasal por 3 meses. A cirurgia foi indicada após a constatação da falha do tratamento clínico. Nessa oportunidade foi aplicado a escala NOSE, ou seja, no pré-operatório após o tratamento clínico. Todos os pacientes incluídos assinaram o Termo de Consentimento Livre e Esclarecido.

As cirurgias foram feitas sob anestesia geral e por um único cirurgião. Em cada paciente somente uma técnica (Turbinectomia endoscópica ou Turbinoplastia) que foi aplicada bilateralmente. Não há na amostra pacientes operados simultaneamente pelas duas técnicas. Não houve distinção patológica entre os pacientes submetidos às duas técnicas.

Tabela I. Escala de Avaliação Sintomática de Obstrução Nasal (NOSE scale).

\begin{tabular}{lccccc}
\hline NOSE & Normal & Leve & Moderado & Acentuado & Severo \\
\hline Congestão nasal & 0 & 1 & 2 & 3 & 4 \\
Obstrução nasal & 0 & 1 & 2 & 3 & 4 \\
Dificuldade de passar o arpelo nariz & 0 & $\mid$ & 2 & 3 & 4 \\
Obstrução nasal ao dormir & 0 & $\mid$ & 2 & 3 & 4 \\
Obstrução nasal aos exercícios & 0 & $\mid$ & 2 & 3 & 4 \\
\hline
\end{tabular}


Os pacientes foram convocados via telefone para uma entrevista em agosto de 2008, com tempo médio de 13,2 meses de pós-operatório. Os entrevistadores foram médicos que não participaram das cirurgias e estavam "cegos" quanto ao tipo de técnica empregada. Em alguns itens foi usada a escala visual analógica (EVA) graduando os sintomas de 0 a 10 conforme a intensidade crescente dos sintomas. Os pacientes foram avaliados quanto aos seguintes itens: escala NOSE pós-operatória (PO), cirurgia associada, morbidade no PO (EVA), uso de tampão, sangramentos, quantidade de crostas no PO (EVA), necessidade de reoperação e uso de medicações no PO tardio.

A seguir os pacientes foram submetidos a nasofibroscopia com fibroscópio Machida ENT-30 PIII de 3,2mm foram analisados quanto aos resultados anatômicos do PO tardio.Exame feito com anestésico tópico e sem vasoconstritor tópico.

\section{Técnica Cirúrgica}

Turbinectomia - Avaliação endoscópica do corneto nasal inferior. Preparo do corneto com solução de vasoconstritor tópico com cloridato de oximetazolina a 0,05\%. A porção do corneto a ser retirada é projetada e clampada com a pinça Rochester. Após cerca de 3 minutos à parte desenhada foi ressecada. A superfície cruenta restante foi tratada com cloridato de oximetazolina a $0,05 \%$ e cauterização com cautério monopolar para controle da hemostasia.

Turbinoplastia - Avaliação endoscópica do corneto nasal inferior. Preparo do corneto com solução de vasoconstritor tópico com cloridato de oximetazolina a 0,05\%. Incisão com lamina de bisturi no 15 . Dissecção do túnel submucoso (entre a mucosa medial e o osso do corneto inferior). Ressecção de parte do osso do corneto e da mucosa lateral. A seguir o retalho lateral foi reposicionado recobrindo a superfície cruenta. A revisão da hemostasia foi feita com cloridato de oximetazolina a 0,05\% e cauterização com cautério monopolar quando necessário.

\section{Critérios de Exclusão}

1) Idade menor que 21 anos;

2) Alterações craniofaciais e/ou mal-formações congênitas;

3) Pacientes com obesidade mórbida;

4) Pacientes portadores de desvios septais com necessidade de correção cirúrgica;

5) Pacientes portadores de polipose nasal e/ou tumores nasais de qualquer espécie;

6) Pacientes portadores de Rinossinusite crônica de qualquer natureza;

7) Pacientes que não concordarem em participar dessa pesquisa e/ou se recusarem a assinar o Termo de Consentimento Livre e Esclarecido.

\section{Análise Estatística}

A análise estatística foi realizada com o software estatístico SPSS para Windows e teste t de Student. O valor de $\mathrm{p}<0,05$ foi considerado estatisticamente significativo.

\section{RESULTADOS}

Todos os pacientes foram contactados por telefone e convocados para entrevista e avaliação otorrinolaringológica. Compareceram para a avaliação 24 pacientes. $\mathrm{O}$ tempo médio de cirurgia foi de 13,2 meses, variando de 8 a 20 meses. Esses pacientes foram divididos em 2 grupos: Grupo I - Pacientes submetidos a turbinectomia inferior com 15 (62,5\%) pacientes;

Grupo II - Pacientes submetidos a turbinoplastia inferior com $9(37,5 \%)$ pacientes.

Os dois grupos foram comparados segundo as variáveis colhidas no pré-operatório, para evitar vieses de confusão. A análise dos grupos se encontra na Tabela 2. A amostra se mostra válida, pois os dois grupos são semelhantes no pré-operatório.

Os dois grupos foram comparados com base nas variáveis colhidas nas entrevistas realizadas no pós-operatório tardio. A principal variável analisada foi à diferença entre a escala NOSE no pré e no pós-operatório tardio. Não houve significância estatística pelo teste t de Student. Os dados são expostos na Tabela 3 .

\section{DISCUSSÃO}

Dentre os fatores analisados na escolha de determinada técnica cirúrgica, o mais importante sem dúvida é a segurança e a experiência do cirurgião.Outros fatores a serem analisados são: a praticabilidade, replicabilidade, grau de morbi-mortalidade e os resultados de estudos clínicos bem controlados e conduzidos. Várias técnicas são descritas na literatura. A via endoscópica é mais segura, pois permite uma melhor avaliação de toda a extensão do corneto nasal, manipulação precisa e hemostasia mais eficiente. As cirurgias com uso de laser e de radiofrequência tem menor morbidade devido ao baixo grau de lesão tecidual, no entanto, são técnicas caras e pouco disponíveis noBrasil.

Turbinectomias e turbinoplastias são as técnicas endoscópicas para o tratamento do corneto inferior mais utilizadas no Brasil. Turbinectomias são tecnicamente mais 
Tabela 2. Comparação estatística das varáveis pré-operatório.

\begin{tabular}{lllc}
\hline & Grupo & Média $\pm \mathrm{DP}$ & $\mathrm{P}$ \\
\hline Idade(anos) & Turbinectomia & $35,53 \pm 12,91$ & 0,737 (ns) \\
& Turbinoplastia & $35,56 \pm 14,30$ & \\
$\mathrm{IMC}^{\mathrm{b}}\left(\mathrm{Kg} / \mathrm{m}^{2}\right)$ & Turbinectomia & $26,47 \pm 2,45$ & $0,41 \mathrm{I}$ (ns) \\
& Turbinoplastia & $22,89 \pm 3,35$ & \\
Tempo de cirurgia(meses) & Turbinectomia & $11,60 \pm 4,03$ & 0,596 (ns) \\
& Turbinoplastia & $16,78 \pm 2,77$ & \\
NOSEPré-operatório & Turbinectomia & $74,00 \pm 17,34$ & 0,923 (ns) \\
& Turbinoplastia & $61,67 \pm 18,37$ & \\
\hline
\end{tabular}

a Desvio-padrão

b Índice de Massa Corpórea

Tabela 3. Comparação estatística dos grupos no pós-operatório tardio.

\begin{tabular}{lllcc}
\hline & CIRURGIA & Média \pm DP & Porcentagem & P \\
\hline VariaçãoNOSE & Turbinectomia & $44,33 \pm 20,16$ & - & 0,237 (ns) \\
& Turbinoplastia & $33,88 \pm 20,73$ & - & \\
Morbidade(EVA $\left.{ }^{2}\right)$ & Turbinectomia & $4,80 \pm 2,65$ & - & 0,32 I(ns) \\
& Turbinoplastia & $3,66 \pm 2,64$ & - & \\
Quantidade de crostas(EVA) & Turbinectomia & $3,80 \pm 2,21$ & - & 0,731 (ns) \\
& Turbinoplastia & $4,00 \pm 2,12$ & - & \\
Sangramento & Turbinectomia & - & $4 / 15(26,6 \%)$ & $0,539(n s)$ \\
& Turbinoplastia & - & $3 / 9(33,3 \%)$ & \\
\hline
\end{tabular}

ascala Visual Analógica - Graduada de 0- 10

b Desvio-Padrão

simples, porem, deixa área cruenta exposta que tem duas consequências principais: a formação de crosta e a necessidade de revisão da hemostasia mais ampla. Teoricamente essa técnica apresenta maior quantidade de crostas e uma maior chance de sangramento. Uma importante vantagem é que a Turbinectomia é mais rápida execução permitindo menor tempo cirúrgico e diminuindo, por conseguinte, a morbidade anestésica.

A Turbinoplastia é uma técnica mais trabalhosa e considerada por alguns mais elegante, tem a vantagem óbvia de não expor área cruenta. Há menos sangramento e menor formação de crostas teoricamente. Conforme o treinamento do cirurgião ela pode ser realizada um tempo satisfatório sem aumentar demasiadamente o tempo cirúrgico.

Os grupos se demonstraram homogêneos no préoperatório. Não houve diferença estatisticamente significante quanto ao IMC, NOSE, idade e tempo de cirurgia. Através de essa seleção procurarmos eliminar alguns vieses de confusão. Como os pacientes tiveram pontuação semelhante na escala NOSE podemos inferir que a morbidade pré-operatória da obstrução nasal nos grupos estava equiparada.
Na avaliação do pós-operatório tardio verificamos que as duas técnicas foram igualmente eficazes na melhora da queixa de obstrução nasal. Ambas tiveram redução importante na escala NOSE, porém sem superioridade significante de uma técnica sobre a outra $(p=0,237)$. As duas técnicas estudadas foram comparadas com relação à quantidade de crostas e morbidade, ambas pela escala visual analógica. Nesses quesitos as duas técnicas se mostraram semelhantes sem diferença estatisticamente significante.

Com relação à incidência de sangramentos no pósoperatório o maior risco teórico da turbinectomia não foi confirmado na nossa amostra. A incidência de sangramentos foi próxima no pós-operatório das duas técnicas. $(\mathrm{p}=0,539)$

Na avaliação dos diversos tipos de tratamento cirúrgico da corneto inferior a literatura mostra resultados semelhantes ao do nosso estudo. Spaci e colaboradores fizeram um estudo comparando turbinectomias por laser, radiofrequência e convencional encontrando resultados semelhantes quanto à melhora da obstrução nasal e a atividade mucociliar (9). Já CAVALIERE comparou turbinectomia tradicional e por radiofrequência achando resultados seme- 
lhantes na melhora objetiva e subjetiva da melhora da obstrução nasal (10).

BHANDARKAR em sua revisão sobre os procedimentos no corneto inferior demonstra que os resultados da cirurgia do corneto inferior são favoráveis e continuam a ser recomendada como um tratamento para hipertrofia de cornetos não responsiva só tratamento clínico. O nível de evidência na literatura está melhorando. Futuros estudos bem desenhados envolvendo coleta de dados prospectiva, as medidas resultantes da validação, análise estatística de comparação, ou grupos de controle e de longo prazo acompanhamento seria fundamental para o nível de evidência (11).

\section{CONCLUSÃO}

Não há evidencia na literatura e em nossa amostra da superioridade da turbinectomia sobre a turbinoplastia e vice-versa quanto à eficácia do tratamento cirúrgico do corneto inferior sobre os outros tratamentos. A escolha da técnica cirurgia deve ser avaliada individualmente em cada indivíduo. Todas as formas avaliadas nesse estudo para o tratamento cirúrgico do corneto inferior são eficazes. O cirurgião deve levar em conta vários fatores como a experiência pessoal, aplicabilidade, replicabilidade e resultados dos estudos científicos para a escolha da técnica cirúrgica adequada.

\section{REFERÊNCIAS BIBLIOGRÁFICAS}

1. Passali D, Passali FM, Damiani V, Passali GC, Bellussi L. Treatment of inferior turbinate hypertrophy: a randomized clinical trial. Ann Otol Rhinol Laryngol. 2003, 112:683-688.

2. Lai VWS, Corey JP. The objective assessment of nasal patency. Ear Nose Throat J. 1993, 72:395-400.

3. Seeger J, Zenev E, Gundlach P, et al. Bipolar radiofrequency-induced thermotherapy of turbinate hypertrophy: pilot study and 20 months follow-up. Laryngoscope. 2001, 113:130-5.

4. Jackson LE, Koch RJ. Controversies in the management of inferior turbinate hypertrophy: a comprehensive review. Plast Reconstr Surg. 1999, 103:300-312.

5. Hol MKS, Huizing EH. Treatment of inferior turbinate pathology: a review and critical evaluation of the different techniques. Rhinology. 2000, 38:157-66.

6. Camelo-Nunes IC, Solé D. Allergic rhinitis: indicators of quality of life. J Bras Pneumol. 2010, 36(1):124-33.

7. Stewart MG, Witsell DL, Smith TL, Weaver EM, Yueh B, Hannley MT. Development and validation of the Nasal Obstruction Symptom Evaluation (NOSE) scale. Otolaryngol Head Neck Surg. 2004, 130(2):157-63.

8. Stewart MG, Smith TL, Weaver EM, Witsell DL, Yueh B, Hannley MT, Johnson JT. Outcomes after nasal septoplasty: results from the Nasal Obstruction Septoplasty Effectiveness (NOSE) study. Otolaryngol Head Neck Surg. 2004, 130(3):283-90.

9. Sapçi T, Sahin B, Karavus A, Akbulut UG. Comparison of the effects of radiofrequency tissue ablation, CO2 laser ablation, and partial turbinectomy applications on nasal mucociliary functions. Laryngoscope. 2003, 113(3):514-9.

10. Cavaliere M, Mottola G, Iemma M. Comparison of the effectiveness and safety of radiofrequency turbinoplasty and traditional surgical technique in treatment of inferior turbinate hypertrophy. Otolaryngol Head Neck Surg. 2005, 133(6):972-8.

11. Bhandarkar ND, Smith TL. Outcomes of surgery for inferior turbinate hypertrophy. Curr Opin Otolaryngol Head Neck Surg. 2010, 18(1):49-53. 